\title{
Looking into Burnout Levels of Freshmen in English Majors of Normal University
}

\author{
Linjing $\mathrm{Xu}^{1, *}$ \\ ${ }^{1}$ Postgraduate of Foreign Languages College, Jiangxi Normal University, China \\ *Correspondence: Postgraduate of Foreign Languages College, Jiangxi Normal University, China. E-mail: \\ xulinjing1993@qq.com \\ This paper is a periodical achievement of the Innovation Fund Project for Graduate Students of Jiangxi Normal \\ University on "Study on the Burnout Levels of English Majors of Normal University and Countermeasure - \\ Illustrated by the Case of Jiangxi Normal University". I appreciate the anonymous referees for the helpful comments \\ and suggestions.
}

Received: November 1, 2017

Accepted: November 17, 2017

Online Published: November 29, 2017

doi:10.5430/wje.v7n6p33

URL: https://doi.org/10.5430/wje.v7n6p33

\begin{abstract}
Nowadays, college students are facing a complex society. Under the influence of various factors, the learning situation of college students has met a variety of problems, among which is learning burnout. The purposes of this study are (1) to assess the burnout levels of freshmen in English majors of normal university; (2) to explore what causes learning burnout and (3) to figure out the possible strategies to reduce the degree of its. The result shows that the means of all responded items is about 2.8286 on average, indicating that the burnout was close to the level of medium. There is no significant difference in terms of gender and only/not only child. However, there is indication of influence of other factors such as knowing little about the major, lack of practice and communication with their seniors.
\end{abstract}

Keywords: learning burnout; English majors; freshmen

\section{Introduction}

Learning burnout is a phenomenon that students have a negative attitude towards school curriculum, which is manifested in physiological, psychological, behavioral and interpersonal communication. In China, Normal Universities are the major institution of training future teachers and important bases of of scientific research. Normal university students are one of the important groups of college students, among which learning burnout may also occur. The English majors are the backbone of the future of national education. Their learning state will affect their learning quality, and in the long run, teaching quality in primary and middle schools when they graduate and become teachers. From this perspective, much attention should be paid to the learning burnout of these students.

\section{Literature Review}

Pines and Katry (1989) first proposed the student burnout, which means the burnout of students in the learning process. According to Maslach (1997), once mentioned that burnout is a popular expression that can easily be used to describe students who have just had some pressure, but it should actually be much more stressful than that. She also believes that burnout is actually a combination of exhaustion, depersonalization, and a negative view of yourself. Because of the important influence of burnout concept of Maslach on burnout research, researchers basically use Maslach's concept with some adjustment to the time and background. For example, Schaufeli (2001) defined the nature of learning burnout as a result of excessive learning demand, the students' emotional exhaustion, alienation from their studies, and low sense of achievement.

Fives and Hamman (2007) have analyzed the burnout problem in student-teaching period. Stoliker and Lafreniere (2015) did the research on the influence of perceived stress, loneliness and learning burnout on University students' educational experience. Kokkinos and Stavropoulos (2016) have studied the burnout phenomenon among teacher trainees. In China, Taiwan scholars began to study burnout earlier, and Zhang (1989) thought that burnout occurred 
at the learning level of students, and they should translate student burnout into learning burnout. The learning burnout is defined as a phenomenon that students in the learning process produce emotional exhaustion, depersonalization and low personal accomplishment because of academic pressure, workload, or other psychological factors. Yang (1998) argued that the above translation can not express the primitive spirit of student burnout, and study burnout should be translated as learning breakdown ,but the concept of learning burnout is still from the Zhang. Lian, Yang and $\mathrm{Wu}$ (2005) developed Burnout Inventory and did a research on college students in mainland China, and put forward that college students learning burnout is students' behavior on account of lack of interest or lack of motivation. Setting in the context of current college students in the China, the study emphasizes the lack of interest in the major, which is the main reason for college students' learning burnout.

In the light of job burnout, Maslach and Jackson (1981) compiled the MBI (Maslach Burnout Inventory). There are three versions of MBI, which consists of three dimensions: emotional exhaustion, depersonalization, and lack of personal accomplishment. The MBI-HSS (MBI-Human Service Survey) is the earliest burnout scale, including 22 items; MBI-Form Ed (MBI-Educators Survey) is based on the original scale and aimed for educators, taking the school organization as the background, including 22 items too. The more recent scale MBI-GS (MBI-General Survey) was developed by Maslach, Jackson and Leiter (1996) for those persons whose jobs are not serving others. The three MBI scale were used in Likert seven self rating scale, in terms of frequency, ranging from "never" to "every day".

In order to measure learning burnout, scholars have revised their scales. For example, Maslach and Schaufeli et al. (2001) had revise the MBI-GB scale, and then made the MBI-SS (Maslach Burnout Inventory-Student Survey) scale. Gold and Michael (1985) did the research on the relationship between academic self-concept and potential burnout in a sample of first-semester elementary school practice teachers. Eventually, they created MBI-CSS (College Student Survey). Taiwan scholar Yang (1989) revised the MBI-GS scale in terms of location, object and work nature to measure the learning burnout of college students in Taiwan. Lian et al. (2005) had taken the actual situation of college students in China into consideration and developed the "learning burnout scale" for college students after a series of research, which includes three dimensions: depression, misconduct, low sense of achievement.

In mainland China, there are a few studies on the learning burnout of students in normal schools, but very few concerns English majors of normal university. They are not only a part of ordinary college students, but also a special group committed to the future national education. In today's social conditions, the status of learning burnout of them, the influencing factors, and other significant problems are all need to be further explored.

\section{Purpose of the Study}

This study was designed to assess learning burnout of freshmen in English majors of normal university which includes exploring the components and dimensions of learning burnout behaviors and analyzing the overall learning burnout problems. The study seeks answers to the following questions:

1) What are the burnout levels of freshmen in English major?

2) What are the factors leading them to feel burnout?

3) What strategies can be adopted in view of the characteristics of learning burnout among them?

\section{Methodology}

\subsection{Participants and Procedure}

Participants were freshmen English majors from a particular class in foreign languages college of a Normal University in southern China. The questionnaires were administered in the class to a total of 40 students. A consent letter was attached on the top of the questionnaire to ensure voluntary and anonymous participation. The students were informed that all the data would remain confidential, and would only be reported by group. The students were permitted to leave at any time during the survey. A total of 35 valid surveys were collected. The effective response rate was $87.5 \%$. Among all respondents, $20 \%$ were males, $80 \%$ females. The valid questionnaires were analyzed using the SPSS 22.0 for statistical analysis.

\subsection{Instruments}

The survey used in this research included two parts; the first part was basic information about school, grades, genders and hometown, the second part was revised version of The Learning Burnout Scale for English majors of normal university. The scale was based upon the Lian Rong Burnout Inventory, with some items revised according to Jiangxi Normal University educational environment to make it suitable for those students. The Learning Burnout 
Scale for English Majors of Normal University is described below.

The Learning Burnout Scale for English Majors of Normal University

Lian Rong Burnout Inventory was based on Maslach Burnout Inventory. The original instrument has shown construct validity and reliability in previous research. Bilge has ever taken 194 college teachers as participants and shoed the Maslach Burnout Inventory has 3 subscales with Cronbach's $\alpha$ coefficients $.830, .650$ and .720 . The scale used in this study were a list of 20 questions, using a Likert five-point scale, with ratings from "always", "sometimes", "not sure", "seldom" to "never", assigned 5,4,3,2 and 1 points respectively so that the higher the score, the stronger the degree of learning burnout.(Q1, 3, 6, 8, 11, 13, 15 and 18 are reverse questions). Among the 20 questions, Q2, 4, 5, 7, 9, 12, 1720 are belong to negative emotional exhaustion; Q1, 8, 10, 14, 16, 19 belong to inappropriate behaviors; Q3, 6, 11, 13,15, 18 are on low sense of achievement.

\section{Results and Discussion}

\subsection{The Result of Questionnaire}

Table 1. Means and the Standard Deviations of Freshmen's Burnout

\begin{tabular}{llll}
\hline & Items & Mean & Standard deviation \\
\hline Learning burnout & Negative emotional exhaustion & 2.7429 & .6466 \\
& Inappropriate behaviors & 3.0190 & .4551 \\
& Low sense of achievement & 2.7238 & .5032 \\
\hline
\end{tabular}

Table 1 shows the means and standard deviations of freshmen's learning burnout. The mean of participant response to all items is 2.8286, indicating that the burnout was close to the level of medium. Among them, the degree of "inappropriate behaviors" is the highest, followed by "negative emotional exhaustion" and "low sense of achievement". Of course, all the means of burnout are between 2.00 and 3.00 points, i.e., between "seldom" to "not sure", especially for the two factors of "negative emotional exhaustion" and "low sense of achievement". Taking 3.00 as the reference, we can know that the learning burnout are "slight".

Table 2. T-test for Genders

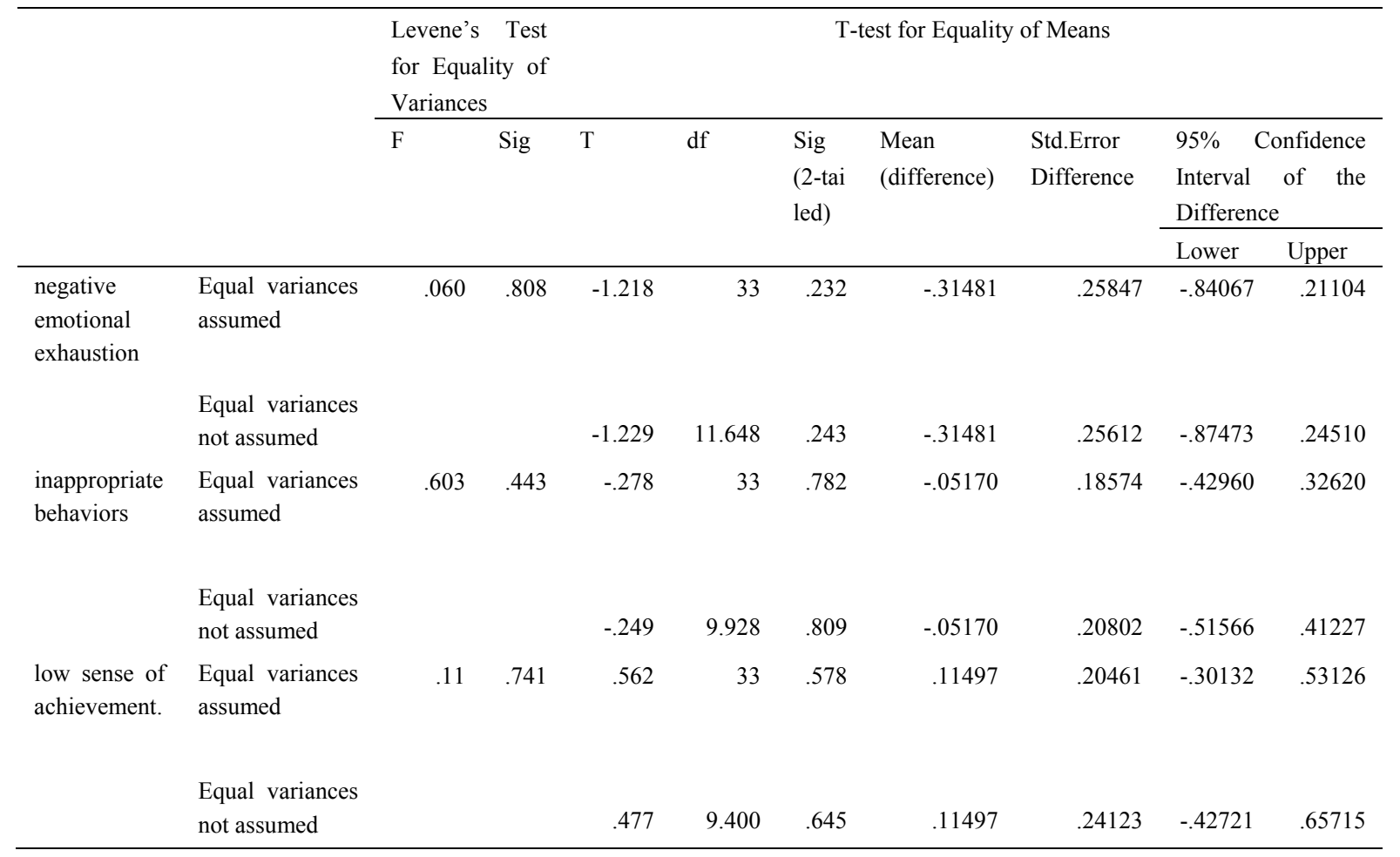


From table 2 , we can see the Sig. of each item is $.808, .443, .741$. They are all bigger than .05 . Therefore, there are no significant deference among genders.

Table 3. T-test for Only/Not Only Child

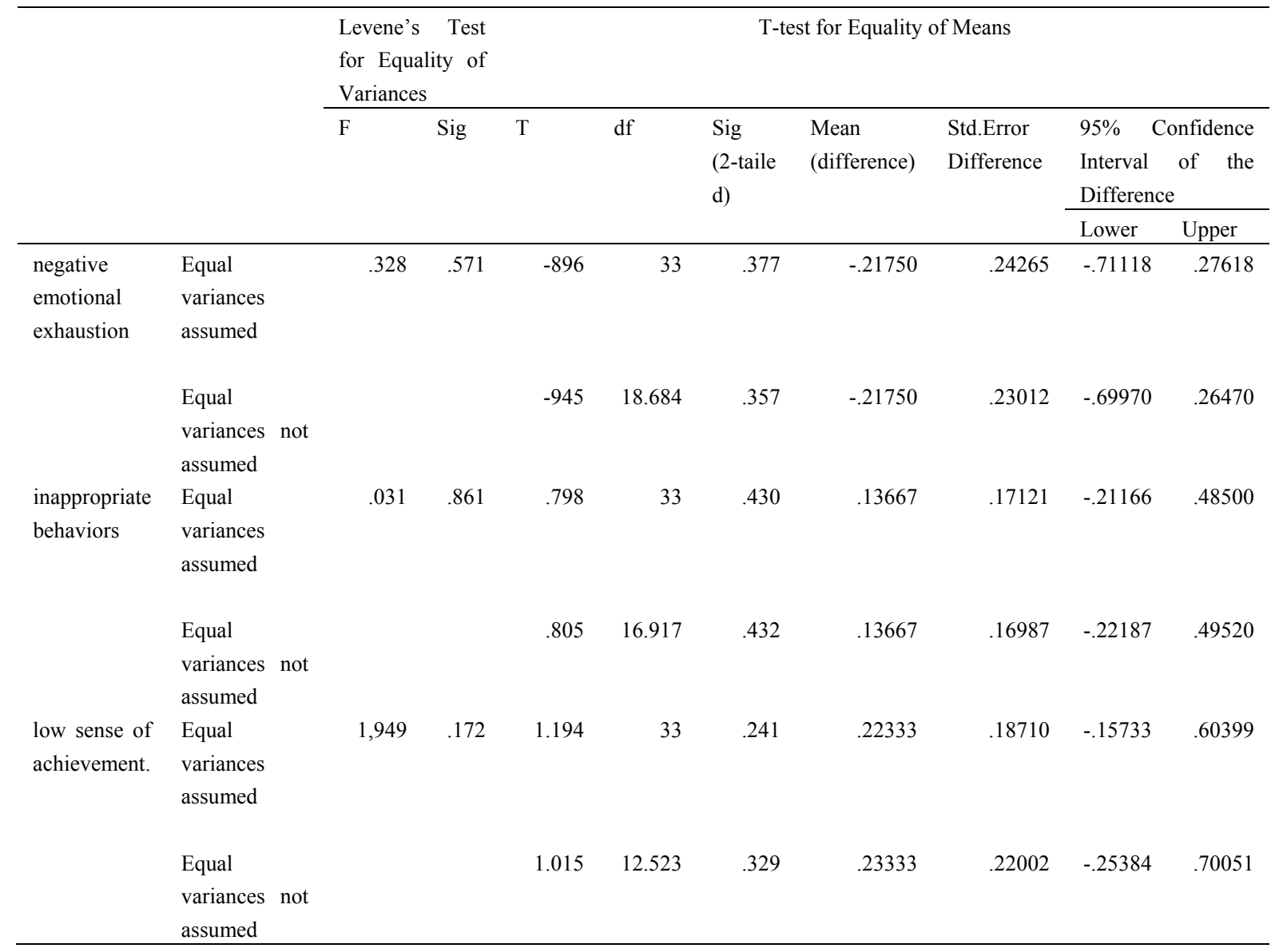

Table 3 shows the Sig. of each item according to their family condition: only child or not. The Sig. of "negative emotional exhaustion" is .571. The Sig. of "inappropriate behaviors", is .816. And the Sig. of "low sense of achievement" is .172 . Those numbers all exceed .05 , so we can draw that only/not only child doesn't make big difference.

\subsection{The Result of Interview}

Apart from the questionnaire method, interview was also used in this study. According to the questionnaire, the means of burnout were between 2.00 and 3.00 points, but it can not mean that there is no learning burnout at all. Through interview, it was found that if they were interested in the courses, they would take part in them positively. If not, they thought taking the class was wasting their time. More than half of the interviewees acknowledge that they could feel nervous and negative about their performance and future. Through the interview, several reasons can be said to be potentially lead to learning burnout. Firstly, students have little information about the major. They liked English indeed, and they wanted to learn English further. That's why they chose English education as their major. However, they seemed be unable to make good connection between English learning and their future career. They thought as English majors they just needed to learn how to speak and write in English well. In fact, those majors are English teachers-to-be. Not only should they enhance their competence in English, but they are also supposed to be good teachers in the future. School authority can not force them to be a teacher. However, they should be responsible for the road they chose. So the difference between facts and imagination was one of the main reasons to learning burnout. Secondly, the curriculum stressed more on theory learning than on the practice. One student says:

"Now that we are learning how to be good English teachers, we should spend more time and energy on practice. If we just read the English articles, listen to VOA or BBC to improve listening skills, recite the 
glossary, we still don't know how to be a teacher. I know that we will take English teaching skills class in one or two year, but I think we should take it now, form the first term. We can practice it in the first three years and take internship in the fourth year. I think that would be better. We can say the theory learning is not important, but we need more practice."

Thirdly, the lack of communication with seniors. Because freshmen don't know much about their training program and how to make a scientific plan, they need to counsel their seniors about it. Sometimes, they don't know what matters most, they want to complete all the missions perfectly. It is very back-breaking and time-consuming. All these can result in learning burnout.

\section{Conclusion}

This study intends to examine the components and situation of learning burnout of freshmen of English majors in Jiangxi Normal University. And from the T-test results above, we know there contains no significant difference in genders and family backgrounds with only child/not only child. Interview, however, reveals there are some other reasons, such as knowing little about the major, lack of practice and communication with seniors.

Based on the findings, we recommend that education officials in Jiangxi Normal University or professionals who are are concerned about education of college students pay more attention to students' learning burnout at the beginning of they college study. As we know, most of universities have established organizations to provide student counseling. School administrators may encourage students to make good use of them. In addition to common courses, school may consider offering more practical courses and opportunities for student to study general education courses. And school can also hold experience exchange salon more frequently to help freshmen become used to it. It should be quite helpful toward reducing the degree of students' learning burnout.

\section{Acknowledgments}

I am very grateful to my supervisor professor Tang, Xiongying, my friends Qin, Yufang and Cheng, Ailian for their helpful comments and suggestions. Thanks all the participants and volunteers in the study.

\section{References}

Fives, H., Hamman, D., \& Olivarez, A. (2007). Does burnout begin with student-teaching? analyzing efficacy, burnout, and support during the student-teaching semester. Teaching \& Teacher Education, 23(6), 916-934. https://doi.org/10.1016/j.tate.2006.03.013

Gold, Y., \& Michael, W. B. (1985). Academic self-concept correlates of potential burnout in a sample of first-semester elementary school practice teachers: A concurrent validity study. Educational and Psychological Measurement, 45, 909-914. https://doi.org/10.1177/0013164485454022

Huang, Y. C., \& Lin, S. H. (2010). Canonical correlation analysis on life stress and learning burnout of college students in taiwan. International Electronic Journal of Health Education, 13, 145-155.

Kokkinos, C. M., \& Stavropoulos, G. (2016). Burning out during the practicum: the case of teacher trainees. Educational Psychology, 36(3), 548-568. https://doi.org/10.1080/01443410.2014.955461

Lian, R., Yang L. X., \&Wu, L. H. (2005). The relationship between College Students' professional commitment and learning burnout and scale development. Journal of psychology, 37(5), 632-636.

Maslach, C., Jackson, S. E., \& Leiter, M. P. (1997). The Maslach Burnout Inventory Manual. Lanham: Scarecrow Press.

Maslach, C., Schaufeli, W. B., \& Leiter, M. P. (2001). Job burnout. Annual Review of Psychology, 52(1), 397-422. https://doi.org/10.1146/annurev.psych.52.1.397

Stoliker, Bryce E., \& Lafreniere, Kathryn D. (2015). The influence of perceived stress, loneliness, and learning burnout on university students' educational experience. College Student Journal, 49(15),146-160.

Yang, H. Z. (1998). A study of factors affecting learning burnout and computer learning outcomes of information management students (Doctoral dissertation, Taiwan national Centre College).

Zhang, Z. Y. (1989). The relationship between locus of control beliefs, social support and learning burnout among College Students. (Doctoral dissertation' Changhua Normal University). 